\title{
Lexical and conceptual processing in Chinese-English bilinguals: Further evidence for asymmetry
}

\author{
HIM CHEUNG and HSUAN-CHIH CHEN \\ Chinese University of Hong Kong, Hong Kong
}

\begin{abstract}
According to the asymmetry model of bilingual representation (Kroll \& Stewart, 1994), the first language (L1) lexicon is closely tied to an underlying conceptual memory, whereas second language (L2) items are mostly associated with their L1 equivalents. An outcome of this architecture is that L1-to-L2, or forward, translation must be mediated by the conceptual memory, whereas L2-to-L1 (backward) translation takes a direct lexical path. Some predictions derived from this hypothetical structure were tested in the present study, which took into account, through analysis of covariance, variations in response production time, concept retrieval time, and some other characteristics associated with the individual test items. Proficient Chinese-English bilinguals were tested on delayed production (Balota \& Chumbley, 1985), picture naming, word translation, and category matching. The expected asymmetrical pattern of translation latencies (i.e., forward > backward) was demonstrated, although it could be statistically explained by the item characteristic of familiarity; matching an L1 item to a category name was faster than matching an L2 item, suggesting relatively strong Ll conceptual links. The present results are best accommodated by a form of asymmetry that allows for nondominant L2-concept linkage, the use of which is conditional upon the familiarity of the test item to the bilingual.
\end{abstract}

Kroll and Stewart (1994) proposed a theoretical framework for the study of the bilingual verbal memorynamely, the asymmetry model, in which communications between first language (L1) and second language (L2) lexicons are achieved via two different pathways. When an item is translated from an L1 to an L2 (i.e., forward translation), the underlying concept is first activated by its $\mathrm{L} 1$ label and then recoded into its $\mathrm{L} 2$ name. This hypothetical route does not assume any direct word-toword links between the two lexicons and regards concept activation as a necessary mediating step for L1-to-L2 communications. When an item is translated from an $\mathrm{L} 2$ to an $\mathrm{L} 1$ (i.e., backward translation), the $\mathrm{L} 2$ label is directly coded into the corresponding $L 1$ name at a lexical level and concept activation is minimally involved. This route exemplifies direct lexical associations between the two lexicons and requires no mediation through concepts in backward translation. The model is, therefore, asymmetrical in the sense that it prescribes qualitatively dif-

This research was supported by a Direct Grant for Research from the Social Science and Education Panel of the Chinese University of Hong Kong and by Earmarked Grants from the Research Grants Council of Hong Kong to H.-C.C. We thank Ching-Yee Chow and Nga-Yan Chan for their assistance in running the experiments. The first author is currently affiliated with the Department of Psychology at the University of Otago, New Zealand. Correspondence concerning this article should be addressed to H.-C. Chen, Department of Psychology, The Chinese University of Hong Kong, Shatin, N.T., Hong Kong (e-mail: ikechen@cuhk.edu.hk).

-Accepted by previous associate editor Kathryn T. Spoehr ferent routes for the two types of translation. The model is depicted in Figure 1.

In Figure 1, the thick solid arrow that points both ways indicates a very strong link between conceptual memory and the $\mathrm{L} 1$ lexicon. The L2-to-L1 thin solid arrow represents the prominent lexical communication, which is of lesser strength, responsible for backward translation; forward translation is accomplished by first going through the strong L1-to-concept route and then the weak concept-toL2 path represented by the dashed arrow. In principle, forward translation can also be achieved at a lexical level through a weak L1-to-L2 link. The model, therefore, identifies lexical/conceptual associations at three different levels of strength, ranging from the strongest, represented by the L1-concept path, to the weakest, realized by the L2-concept link. Since the three constructs of an L1 lexicon, an L2 lexicon, and conceptual memory are fully connected with one another, the model's emphasis on the lexical and conceptual nature of backward and forward translation, respectively, is by no means absolute. Alternative pathways could be employed, when necessary. The model simply formalizes what the bilingual tends to do in normal situations, not what he or she must do under all possible conditions.

Owing to its unique architecture, the asymmetry model predicts shorter latencies for backward than for forward word translation, since the former does not normally require concept access, whereas the latter usually necessitates the activation of both an L1-to-concept and a concept-to-L2 link. This expected asymmetrical pattern of word translation latencies is exactly what has been 


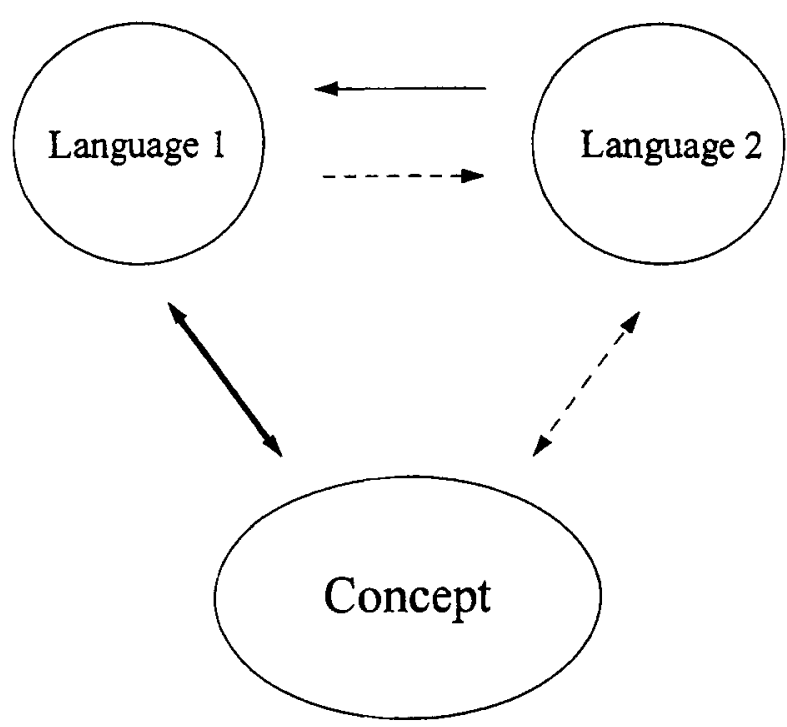

Figure 1. A schematic representation of the asymmetry model of bilingual memory. From "Category Interference in Translation and Picture Naming: Evidence for Asymmetric Connections Between Bilingual Memory Representation," by J. F. Kroll and E. Stewart, 1994, Journal of Memory \& Language, 33, pp. 150. Copyright 1994 by Academic Press. Adapted with permission.

found (see, e.g., Kroll \& Curley, 1988; Kroll \& Stewart, 1994). Additional support for the model has to do with the sensitivity of the translation process to semantic variables. Kroll and Stewart reported that semantically organized word lists had a negative effect on translation latencies in forward but not in backward translation, indicating that only the former might have involved concept activation and, thus, was sensitive to semantic organization. Using a different paradigm, Sholl, Sankaranarayanan, and Kroll (1995) found in a recent study that, if concepts were repeated across picture naming and word translation, positive transfer in response latencies occurred with forward but not with backward translation. This finding must be taken as evidence for disparity in conceptual processing between the two translation conditions, for such transfer of training could only take place at a semantic level.

Other studies, however, stress the similarity rather than the difference between the two translation directions and demonstrate predominant conceptual processing for both. La Heij, Hooglander, Kerling, and van der Velden (1996) reported significant Stroop facilitatory effects, for both forward and backward word translation, indicating qualitatively similar conceptual processing for the two lexicons. Indeed, it has been shown in a number of previous studies using different paradigms that the L2 lexicon has prominent, direct associations with the conceptual memory, just as does the L1 lexicon (see, e.g., Altarriba, 1990; Chen, 1990; Chen \& Ho, 1986; Chen \& Ng, 1989; Keatley, Spinks, \& de Gelder, 1994). The question, hence, is not whether certain translation pathways exist; rather, it becomes under what conditions the activation of a particular set of routes is especially encouraged and in what situations it is not.

As a result, more recent development in the conceptualization of the asymmetry model has added on to the prototypical version some extra dimensions that actually condition bilingual lexical/conceptual processing. These constraints further strengthen the predictive power of the model. The work of Dufour and Kroll (1995) provides an example of such endeavor. The authors investigated the critical component of strength of conceptual activation associated with both $\mathrm{L} 1$ and $\mathrm{L} 2$ items and manipulated in their study the factor of L2 fluency. For a group of more fluent and a group of less fluent English-French bilinguals, a category-matching task was used in which the subject was to decide as quickly as possible if a target concept was a member of a certain category. Both the target and the category name varied in presentation language (English vs. French). Results showed a clear dissociation of the pattern of matching latencies between the two subject groups. The more fluent bilingual was insensitive to the category name language and generally performed better with English than with French target names. The less fluent bilingual, on the other hand, performed better when the language of the category name matched that of the target name. It was concluded that, for the more fluent bilinguals, both English and French words activated similarly rich semantic representation, and, hence, language of the category name did not make a difference in matching performance. For the less fluent bilinguals, however, French words activated significantly less semantic information than did English words, and such discrepancy manifested itself as an inhibitory effect when there was a mismatch of target and category name language in an experimental trial: An English word activated a number of semantic nodes that were not shared by the corresponding French word, and the activation of these irrelevant nodes interfered with matching performance. Such interference simply did not occur with (1) proficient, or balanced, bilinguals who had similar activation levels for English and French words, so that very few irrelevant nodes were activated even when there was a language mismatch; (2) less fluent bilinguals when the category and the target name were in the same language, so that no irrelevant nodes were active. The interpretation offered by Dufour and Kroll qualifies the asymmetry model by putting on the constraint that L2 proficiency correlates positively with the strength of $\mathrm{L} 2$-concept associations; it is in line with the findings of some previous studies that also take a developmental perspective (see, e.g., Altarriba \& Mathis, 1997; Kroll, Dufour, Sholl, \& Roufca, 1993; Talamas \& Kroll, 1993; see also Kroll \& Sholl, 1992).

In addition to the individual characteristic of L2 proficiency, effects of various stimulus attributes on the translation process have also been explored. In her first two experiments using the tasks of Dutch-to-English (forward) word translation and translation recognition, 
de Groot (1992a) reported that both the occurrence frequency and imageability of the Dutch stimulus words affected word translation and translation recognition latencies. Word imageability was operationalized as the easiness of generating concrete mental pictures/images when a certain test word was encountered. It was reported that both high-frequency and high-imageability words produced shorter translation latencies than did lowfrequency and low-imageability ones. In another experiment using the multiple regression paradigm, the author identified four word characteristic variables, each of which uniquely predicted word translation latencies: frequency of stimulus words, frequency of response words, context availability (i.e., the easiness of coming up with specific contexts in which what the test word denoted tended to appear), and cognate status of translations (i.e., whether the test word and its target translation were similar in spelling and sound). These variables were negatively correlated with translation latencies. To interpret the findings, de Groot (1992a) proposed a hierarchical architecture with word-to-concept and word-to-word connections of specific strengths. The conceptual memory underlying the lexicons was conceptualized in a distributed manner (see also de Groot, 1992b). Test words that measured high in the critical characteristics activated especially numerous semantic nodes, and this enhanced the speed of linking up with target translations. Translation latencies were therefore shortened.

In the same vein, de Groot, Dannenburg, and van Hell (1994) examined the effects of various word characteristic variables on both forward and backward translation. Meaning (semantic) variables, such as imageability, context availability, and definition accuracy (i.e., how easily and accurately a test word could be verbally defined) associated with both the stimulus and the response words, were found to be correlated with both forward and backward translation latencies, but somewhat more strongly in the forward than in the backward condition. Furthermore, the differential effects of the semantic variables on the two translation tasks were qualified by the cognate status of translations: For noncognates (test and target words looked and sounded different), the correlation between latency and the semantic variables was higher in the forward than in the backward condition; for cognates (test and target words looked and sounded similar), no directionality effect on the correlation, which was comparable to that for noncognates in backward translation, was observed. It was argued that, when the translations were cognates, their similar orthographies and phonologies (lexical level characteristics) encouraged the use of a direct lexical path, regardless of translation direction, whereas, for noncognates, the use of the lexical path was not particularly encouraged by any lexical level similarities between the test and the target words, and, therefore, word translation displayed the usual asymmetrical pattern. The findings reported by both de Groot (1992a) and de Groot et al. (1994) are generally compatible with the asymmetry model in that forward translation appears to be more sensitive to semantic variables than is backward translation; they also indicate that stimulus attributes affect translation latencies, probably by constraining the activation of the different routes postulated by the model during translation.

Using the asymmetry model as a framework, the present study attempts to look systematically into the functioning of the various pathways interconnecting the two lexicons and the conceptual memory in proficient ChineseEnglish bilinguals. Most previous studies in the area have examined languages belonging to the same familynamely, the Indo-European language family. For instance, Altarriba and Mathis (1997) used native English speakers who had learned Spanish; La Heij et al. (1996) recruited Dutch-English bilinguals; Kroll and Curley (1988) looked at English and German, whereas Dufour and Kroll (1995) used English-French bilinguals. These languages are all closely related, and they do have a number of lexical level similarities. Hence, one is not sure whether conclusions generated in these studies would apply to bilinguals speaking two virtually unrelated languages. It has been documented that the Chinese language has a number of unique characteristics that clearly distinguish it from English in terms of cognitive processing. Experimentally, for instance, it has been demonstrated that lexical decision is generally faster than naming in Chinese, whereas the reverse is true in English (see Chen, 1992, 1996, for more detailed discussions). Orthographic factors have been suggested to account for such discrepancy (Chen, 1996). The need to replicate bilingual findings based on closely related European tongues with the distinctive languages of English and Chinese is, therefore, obvious for the generalizability of the asymmetry model. Potter, So, von Eckardt, and Feldman (1984) did use Chinese-English bilinguals, but they did not directly test the model. To evaluate the universal applicability of the asymmetry model with Chinese and English therefore constitutes a major motivation of the present study.

Another objective of this research is to further explore the effects of certain item attributes on bilingual processing that have not been sufficiently studied by workers in the area. In a sense, the current study attempts to replicate some of de Groot's (1992a) and de Groot et al.'s (1994) findings in order to obtain converging evidence for, and thus reinforce, the notion that bilingual lexical/ conceptual processing is conditional upon certain test item characteristics. The present study, however, departs from the work of de Groot and her colleagues in that a different approach (i.e., the analysis of covariance [ANCOVA] procedure) and a different population (i.e., proficient Chinese-English bilinguals) are used. Picturenaming and word translation latencies were used to estimate the extent to which a particular set of bilingual processing routes was active; extra information about the individual test items was also obtained. First, the test item characteristics of familiarity, imageability, context availability, occurrence frequency, and number of phonemes were coded and used as covariates in a series 
of ANCOVAs. Details of communications among the two lexicons and the conceptual memory (as revealed by picture naming and word translation) could thus be examined both before and after the item characteristic variables were equated across experimental conditions (i.e., adjusted to zero by the ANCOVA procedure). Second, the tasks of delayed production and category matching were used to estimate response production and concept retrieval latencies, respectively, for individual test items, and the estimates were used as covariates in another set of ANCOVAs. This was to examine patterns of internal route activation both before and after response production, and concept retrieval latencies for the test items were equated across experimental conditions.

The mentioned procedures may need some elaboration. In examining patterns of communication among the lexicons and the conceptual memory, the present study contrasted word translation with picture naming, on the one hand, and forward with backward translation, on the other (see Kroll \& Stewart, 1994; Potter et al., 1984). As Snodgrass (1984) has argued, drawing conclusions about internal processes through comparisons among different tasks makes the assumption that these tasks do not differ from one another in processes other than those in question. For instance, comparing forward to backward translation latencies for information about the internal translation process would obviously require the equation of response production across the two translation conditions. Response language, however, varies systematically across the two conditions (forward and backward translation require $\mathrm{L} 2$ and $\mathrm{L} 1$ responses, respectively); it is likely that $\mathrm{L} 1$ and $\mathrm{L} 2$ production latencies are inherently different, independent of any internal translation processes. Similarly, comparing, say, L1 picture naming with backward translation latencies for information about internal processing paths would require the equation of concept access (retrieval) speed across the two tasks. Nevertheless, $\mathrm{L} 1$ picture naming always has picture stimuli, whereas backward translation uses L2 stimulus items, and these two types of stimuli may simply have very different concept retrieval speeds. The present study, hence, used delayed production (Balota \& Chumbley, 1985) to estimate response production latencies and category matching to estimate concept retrieval times associated with individual test items. These estimates were used as covariates so that internal translation processes could be examined both before and after their effects were taken into account. The use of item familiarity, imageability, context availability, occurrence frequency, and number of phonemes as covariates in another set of ANCOVAs was based on previous findings that they were correlated with the translation process (de Groot, 1992a; de Groot et al., 1994).

There were four hypotheses derived from the asymmetry model and tested in the present study. First, forward translation would be slower than backward translation, because the former involved both concept activation and
L2 labeling, whereas the latter required only activating the appropriate lexical path. Second, L1 picture naming would be faster than backward translation, because $\mathrm{Ll}$ picture naming is accomplished via a very strong $\mathrm{L} 1-$ concept link, whereas the lexical route used in backward translation is relatively weak (see Figure 1). Third, L2 picture naming would be almost as slow as forward translation, because the former is based on a weak L2concept path and the latter requires both concept access and L2 labeling. Fourth, matching an L1 item to a category name would be faster than matching an $\mathrm{L} 2 \mathrm{item}$, due to strong Ll-to-concept links.

The present study consisted of three experiments. Experiment 1 tested the first three hypotheses with stimuli matched in recognition threshold across conditions. Experiment 2 had independent subjects rate all verbal test items on their familiarity, imageability, and context availability and all picture test items on their familiarity and context availability. These ratings were used together with occurrence frequencies and number of phonemes for verbal items as covariates in a series of ANCOVAs that retested the first three hypotheses. In Experiment 3, delayed production and category-matching latencies were obtained for the test items and used as covariates to reevaluate the same three hypotheses. The fourth prediction was also tested with category-matching data.

\section{EXPERIMENT 1}

Experiment 1 tested a group of proficient ChineseEnglish bilinguals on picture naming and word translation. Comparisons of latencies among these tasks potentially reveal the bilingual memory structure (Potter et al., 1984). The present hypotheses, according to the asymmetry model (Kroll \& Stewart, 1994), were that (1) forward translation would be slower than backward translation; (2) naming pictures in $\mathrm{L} 1$ would be faster than backward translation; and (3) naming pictures in L2 would take as long as forward translation. Test items matched in recognition threshold were used.

\section{Method}

Subjects. Thirty-six undergraduates at the Chinese University of Hong Kong, who were proficient Chinese-English bilinguals, participated in Experiment 1. These subjects had been learning English formally in the classroom for at least 12 years at the time of testing. All the subjects reported normal or corrected-to-normal eyesight.

Materials and Apparatus. Forty pictures and their corresponding Chinese and English written names, totaling 120 items, were used as stimuli. The 40 pictures had been experimentally selected from Snodgrass and Vanderwart's (1980) standardized picture set in an earlier study done in our laboratory (Chen, Cheung, \& Lau, 1997). The principle of selection was that mean recognition thresholds across the three forms of picture, Chinese word, and English word for any given picture-concepts should be of least discrepancies. In each selection trial, the subject was instructed to name as quickly as possible a visually presented stimulus, which could be a picture, a Chinese word, or an English word. Pictures and Chi- 
nese words required Chinese responses, whereas English words required English responses. The stimulus was initially presented for $20 \mathrm{msec}$, followed by a 1.5 -sec mask. If the subject did not respond, the stimulus would be presented again with a $10-\mathrm{msec}$ increase in presentation duration. The sequence was repeated until the stimulus was correctly named or until the presentation duration exceeded 120 msec. A Gerbrands T-4A four-field tachistoscope was used for stimulus presentation. As a result, 80 pictureconcepts were selected, with mean recognition thresholds (in milliseconds) of $49.1,40.1$, and 47.0 for the stimulus types of picture, Chinese word, and English word, respectively; the corresponding error rates were $7.7,2.5$, and 3.0. The maximum between-stimulus type difference in threshold was only $9 \mathrm{msec}$ (cf. Potter et al., 1984, who reported that the recognition threshold for pictures was $22 \mathrm{msec}$ higher than that for Chinese words). The 40 picture-concepts used in the present experiment were randomly selected from these 80 concepts.

Stimuli were displayed on an 80486 personal computer in Experiment 1 . Areas of the displays were approximately equal for pictures, Chinese words, and English words. The computer was connected to a voice detection key that stopped the timer of the computer upon detecting a vocal response.

Procedure. Experiment 1 consisted of two blocks (Chinese and English) of 10 picture-naming trials and two blocks (forward and backward) of 10 word translation trials. Each block was preceded by 2 practice trials. Concepts within each block were presented in the same order. The order of block presentation was counterbalanced across subjects. Instructions were given orally by the experimenter before each block. No concept was repeated in any form for any subject. To begin a picture-naming trial, a square of arrays of small crosses was displayed for $1 \mathrm{sec}$ to serve as a mask and a starting signal. It was followed by a picture stimulus that stayed on the monitor until the subject responded. The subject was instructed to respond by saying the name of the picture out loud as quickly and accurately as possible. No feedback was given. If the subject did not know the answer, he or she had to give the vocal response "skip." The experimenter sat beside the subject throughout the experiment and recorded the missing trials. Naming latency was defined as the time interval between the onset of the picture stimulus and the onset of the subject's vocal response, which was picked up by a voice detection key. The experimental procedure of word translation was the same as picture naming, except that words instead of pictures were presented and translations rather than names of pictures were required.

\section{Results and Discussion}

Percent error. Error rates in the four experimental conditions are presented in Table 1.

Driven by the hypotheses, contrasts between forward and backward translation, $\mathrm{L} 1$ picture naming and backward translation, and L2 picture naming and forward translation were tested. The only significant contrast was that between forward and backward translation $[t(78)=$ $2.28, p=.026]$ indicating a greater percent error in forward than in backward translation.

Latencies: Analyses by subject. Mean response latencies in the various conditions were calculated, using the subject as the unit of analysis. They are presented in Table 1. First, forward translation was significantly slower than backward translation $\left[F(1,35)=6.35, M S_{\mathrm{e}}=34,052.6\right.$, $p=.016]$. This result is consistent with the claim of asymmetry, which specifies more processing stages for forward than for backward translation. Second, L1 picture
Table 1

Mean Picture Naming and Word Translation Latencies (in Milliseconds; With Percent Error) for Experiment 1: Analysis by Subject

\begin{tabular}{|c|c|c|c|c|}
\hline \multirow[b]{3}{*}{ Task } & \multicolumn{4}{|c|}{ Response Language } \\
\hline & \multicolumn{2}{|c|}{ Chinese } & \multicolumn{2}{|c|}{ English } \\
\hline & $M$ & $\%$ & $M$ & $\%$ \\
\hline Picture naming & 992 & 5.0 & 1,241 & 13.6 \\
\hline Word translation & 1,239 & 9.4 & 1,348 & 16.7 \\
\hline
\end{tabular}

naming was faster than backward translation $[F(1,35)=$ $\left.68.04, M S_{\mathrm{e}}=16,078.5, p<.001\right]$. This result confirms the hypothetical contrast between a very strong L1concept link and a prominent but weaker L2-to-L1 direct lexical link specified by the model. Third, naming pictures in L2 took about as long as translating $\mathrm{L} 1$ items into their $\mathrm{L} 2$ equivalents $\left[F(1,35)=3.02, M S_{\mathrm{e}}=44,952.9\right.$, $p>.05]$. This finding is compatible with the claim that forward translation involves both detailed conceptual processing and L2 labeling and is, thus, as slow as L2 picture naming, which is based on a weak conceptual path.

Latencies: Analyses by item. Mean response latencies were also calculated using the test item as the unit of analysis. Forward translation, again, was slower than backward translation $\left[F(1,78)=4.47, M S_{\mathrm{e}}=80,854.1, p<.04\right]$. This result is compatible with the asymmetry claim, which specifies more processing stages for forward than for backward translation. $L 1$ picture naming was faster than backward translation $\left[F(1,78)=29.11, M S_{\mathrm{e}}=41,364.7\right.$, $p<.01$ ]; naming pictures in L2 took about as long as translating $\mathrm{L} 1$ items into their $\mathrm{L} 2$ equivalents $[F(1,78)=$ $\left.2.02, M S_{\mathrm{e}}=98,650.1, p>.10\right]$. The pattern of results is identical to that based on the individual subjects.

Using stimulus items with very similar recognition thresholds across experimental conditions, Experiment 1 obtained data supportive of the asymmetry model. Both analyses by subject and analyses by test item revealed that forward translation was slower than backward translation, suggesting that verbal items in the two lexicons were differentially processed. Forward translation was also more error-prone than backward translation. The fact that bilinguals speaking two very different languages (one Indo-European and one East Asian) were examined in the present study further adds to the universal applicability of the asymmetry model, as evidence for the model has by and large been based on closely related European tongues. Communications from the $\mathrm{L} 1$ to the $\mathrm{L} 2$ lexicon appeared to be conceptually based (i.e., requiring both concept activation and L2 labeling), for forward translation was as slow as L2 picture naming, which relied on a weak L2-concept path. L1 picture naming was faster than backward translation, thus confirming the hypothetical contrast between a strong LI-concept tie and a relatively weak L2-to-L1 lexical link. Overall, the present results do suggest a form of asymmetry that specifies 
(1) different internal processing stages for L1 and L2 items and (2) lexical/conceptual associations of different strengths.

\section{EXPERIMENT 2}

As shown by de Groot et al. (1994), certain item level characteristics are correlated with the processing of lexical items to a significant extent. Although exactly how these characteristics affect lexical processing is not well understood, it is reasonable that their effects should be taken into consideration when interpreting data on various lexical processing mechanisms. Experiment 2 obtained measures of some item characteristics associated with the stimuli used in the preceding experiment, using them as covariates in a series of ANCOVAs that reanalyzed the picture-naming and word translation data of Experiment 1 and retested the three hypotheses. Subjective ratings from an independent group of proficient ChineseEnglish bilinguals on the familiarity, imageability, and context availability of the verbal test items, as well as their occurrence frequencies and number of phonemes, were obtained. For the picture stimuli, familiarity and context availability ratings were gathered. This experiment departed from the work of de Groot et al. and would thus add to it because (1) this experiment examined the distinctive languages of Chinese and English, the interactions between which might be very different from those among related European languages, and (2) this experiment used the ANCOVA method, which allowed direct evaluation of the asymmetry model and statistical control of item attributes at the same time.

\section{Method}

Subjects. Twelve undergraduate and graduate students at the Chinese University of Hong Kong were asked to rate the test items used in the preceding experiment on certain characteristics. These subjects were all proficient Chinese-English bilinguals who had been learning English as a second language formally for at least 12 years at the time of testing. Their general language background and L2 (English) proficiency were very much comparable with those of the Experiment 1 subjects because: (1) the two subject groups had undergone the same language curriculum in high school; (2) they had, by and large, the same language experience at home in terms of the predominantly monolingual environment of spoken language (i.e., Cantonese-Chinese); and (3) they had been required to demonstrate through examination a certain level of English proficiency before they could enroll at the university. All the subjects in the present experiment reported normal or corrected-to-normal eyesight. None of them had participated in Experiment 1

Materials. The same 120 stimulus items as those used in Experiment 1 (i.e., 40 picture-concepts, together with their Chinese and English written names) were again used in the present experiment.

Procedure. The subjects were asked to rate the 120 items on the following characteristics by using a 5-point scale.

Familiarity (both words and pictures). This was defined as the number of times the subject had experienced the item (de Groot et al., 1994; Noble, 1953), with $1=$ never seen, heard, or used it before and $5=h e /$ she had seen, heard, or used it almost every day.
Imageability (words only). This was defined as how easily and quickly it was possible to come up with a mental image in response to the item (de Groot et al., 1994; Paivio, Yuille, \& Madigan, 1968), with $1=$ very difficult and slow and $5=$ very easy and quick.

Context availability (both words and pictures). This was defined as how easy it was to produce contexts or circumstances in which the item might appear (de Groot et al., 1994; Schwanenflugel, Harnishfeger, \& Stowe, 1988), with $1=$ very difficult and $5=$ very easy.

Instructions were presented to the subject both orally by the experimenter and in printed form. The language of instruction was Chinese, with which the present subjects were more familiar. It was stressed that what should be rated were characteristics of the words or the picture-images themselves, not the concepts that they represented.

In addition to the mentioned characteristics, the variables of occurrence frequency and number of phonemes were also defined for the Chinese and English items. Frequencies of the English words were obtained from Francis and Kučera (1982) and those of the Chinese items from $A$ Frequency Count for Words Commonly Used by Hong Kong Junior Secondary School Students (Hong Kong Department of Education, 1986).

\section{Results and Discussion}

Because the covariates (i.e., the item characteristic measures) obtained in the current experiment were tied to the individual test items, performance of an ANCOVA was possible only with the test item as the unit of analysis. All subsequent analyses are, therefore, based on individual test items. Means for the various item characteristic variables are presented in Table 2.

One-way ANOVAs were performed in order to examine differences in these measures among picture, Chinese, and English stimuli. Significant differences were found only for context availability $\left[F(2,117)=12.27, M S_{\mathrm{e}}=\right.$ $0.16, p<.001]$ and occurrence frequency $[F(1,78)=4.81$, $\left.M S_{\mathrm{e}}=8,972.6, p=.03\right]$. For context availability, both the contrast between pictures and Chinese words $[F(1,78)=$ 23.87, $\left.M S_{\mathrm{e}}=0.16, p<.001\right]$ and that between pictures and English words $\left[F(1,78)=11.57, M S_{\mathrm{e}}=0.13, p=\right.$ $.001]$ were significant. These analyses showed that the presently used English test words were more frequent than their Chinese translations; the picture stimuli were more easily put into contexts than both the Chinese and the English words.

Retesting the hypotheses of Experiment 1. Measures of the item characteristics were used as covariates in a set of ANCOVAs that reanalyzed the picture-naming and word translation data of Experiment 1, using the test

Table 2

Means for the Item Characteristic Variables (With Standard Deviations) in Experiment 2

\begin{tabular}{|c|c|c|c|c|c|c|}
\hline \multirow[b]{2}{*}{ Variable } & \multicolumn{2}{|c|}{ Pictures } & \multicolumn{2}{|c|}{ Chinese } & \multicolumn{2}{|c|}{ English } \\
\hline & $M$ & $S D$ & $M$ & $S D$ & $M$ & $S D$ \\
\hline Familiarity & 4.5 & 0.2 & 4.4 & 0.4 & 4.5 & 0.3 \\
\hline Context availability & 4.4 & 0.3 & 4.0 & 0.5 & 4.1 & 0.4 \\
\hline Imageability & & & 4.3 & 0.3 & 4.4 & 0.2 \\
\hline Number of phonemes & & & 4.2 & 1.6 & 3.9 & 1.8 \\
\hline Frequency & & & 28.0 & 56.1 & 74.5 & 121.7 \\
\hline
\end{tabular}

Note - Frequency = occurrence frequency per one million words; all measures other than frequency are on a 5-point scale. 
item as the unit of analysis (simple correlations between picture naming, word translation latencies [from Experiment 1], and the relevant item characteristic variables are shown in Appendix A). The reanalyses were driven by the previously tested hypotheses. First, it had been hypothesized that forward translation would be slower than backward translation. This comparison involved only word stimuli, and, therefore, all the variables were entered as covariates. The five covariates together accounted for a significant portion of the translation latency variance $\left[F(5,72)=4.41, M S_{\mathrm{e}}=64,731.5, p=.001\right]$. The only significant covariate was familiarity $[t(72)=-2.68$, $p=.009]$. After the adjustment, forward translation was no faster or slower than backward translation $[F(1,72)=$ $\left.2.18, M S_{\mathrm{e}}=64,731.5, p=.14\right]$, with adjusted means of $1,345.1$ and $1,257.0$, respectively. The first hypothesis derived from the asymmetry model was not supported.

Second, it had been hypothesized that L1 picture naming would be faster than backward translation. This comparison involved both word and picture stimuli, and, thus, only the covariates of context availability and familiarity were used. The two covariates together accounted for a significant portion of the naming/translation latency variance $\left[F(2,76)=10.34, M S_{\mathrm{e}}=33,370.1, p<.001\right]$. Nevertheless, only familiarity was a significant covariate $[t(76)=-3.42, p=.001]$. After the adjustment, $\mathrm{L} 1$ picture naming was still faster than backward translation $\left[F(1,76)=26.79, M S_{\mathrm{e}}=33,370.1, p<.001\right]$, with adjusted means of $1,000.9$ and $1,233.3$, respectively. The second hypothesis was supported.

Third, it had been hypothesized that L2 picture naming would be no faster or slower than forward translation. This comparison involved both word and picture stimuli, and, therefore, only the covariates of context availability and familiarity were used. The two covariates together accounted for a significant portion of the naming/translation latency variance $\left[F(2,76)=8.0, M S_{\mathrm{e}}=\right.$ $83,840.5, p=.001]$. Nevertheless, only familiarity was a significant covariate $[t(76)=-2.66, p=.01]$. After the adjustment, L2 picture naming was no faster or slower than forward translation $\left[F(1,76)=0.28, M S_{\mathrm{e}}=83,840.5, p=\right.$ $.60]$, with adjusted means of $1,301.8$ and $1,344.3$, respectively. The third hypothesis was supported.

The only change of results from the preceding to the present experiment, which measured certain item characteristics and used them as covariates in the reanalyses, was that the two word translation tasks no longer displayed different response latencies. The straightforward interpretation is that the difference in translation time found in Experiment 1 could possibly be explained by item familiarity, independent of any processing asymmetries. Nevertheless, the present ANCOVAs only use the test item as the analysis unit, and caution must therefore be taken not to overinterpret the results. What the present findings suggest is an alternative way to interpret the translation time asymmetry so frequently observed in previous studies (which did not control for variations in test item familiarity); the activation patterns of the translation routes specified by the asymmetry model may actually be conditional upon the familiarity of the test items. Future research in the area should, therefore, (1) attempt some form of control over item familiarity so as to render the data more interpretable and (2) explore in a more concrete way how item familiarity affects patterns of communication among the lexicons and the conceptual memory.

\section{EXPERIMENT 3}

The aim of Experiment 3 is twofold. (1) It evaluates the proposition that the $\mathrm{L} 1$ lexicon has stronger conceptual links than the L2 lexicon. (2) Using an ANCOVA, it retests the hypotheses of the two preceding experiments by introducing response production and concept retrieval latencies as covariates. Derived from the first objective is the new hypothesis that matching an $\mathrm{Ll}$ item to a category name would be faster than matching an L2 item, assuming that strong conceptual links facilitate concept access and, therefore, category-matching performance. The second objective was motivated by the analysis of picture-naming and word translation latencies into various components. Some of these components, such as the time needed for producing overt responses and the time needed for accessing concepts in response to stimuli, are irrelevant to the translation pathways specified by the asymmetry model. For instance, the contrast between forward and backward translation times involves comparing a latency containing $L 2$ response production time (i.e., forward translation latency) with a latency containing $\mathrm{L} 1$ response production time (i.e., backward translation latency). Since response production time is irrelevant to the issue under examination, systematic variations in it should be controlled in some form. A similar problem arises when picture-naming latency is compared against word translation time, for the former contains concept retrieval time in response to a picture, whereas the latter contains concept retrieval time in response to a word, and it is conceivable that there are systematic but extraneous variations in the time needed for concept access due to different stimulus types (pictures vs. words). Possible variations in response production and concept access times have not been controlled by previous studies. On the basis of the same test items as those used in Experiment 1 but a different group of proficient ChineseEnglish bilinguals, the present experiment employed the tasks of delayed production and category matching to estimate each test item's response production and concept retrieval time, respectively.

The rationale for using delayed production to indicate sheer vocal response latency was that processing stages preceding response production, such as stimulus recognition and phonological code access, would have been completed by the end of the 650 -msec delay (i.e., the interval between stimulus onset and response signal) used in the present experiment. Balota and Chumbley (1985) used the same task with the same delay and obtained sta- 
ble results. Category matching was used in the present study to indicate concept retrieval time. Categories of different broadness were used, because matching a certain target to a category name might not require full activation and precise knowledge of the concept underlying that target. To judge whether an item belonged to a certain category might only need information about some particular aspects of the item, such as animacy and mobility, depending on the broadness of the category name. It was, therefore, important to use categories of different broadness, which supposedly demanded different levels of concept activation, to examine the concept retrieval process. Empirically, Jared and Seidenberg (1991) did demonstrate that categories of different broadness behaved differently in a semantic decision task.

In the present experiment, estimates obtained from both delayed production and category matching were used as covariates in a series of ANCOVAs that reanalyzed the picture-naming and word translation data obtained in Experiment 1 and retested the three hypotheses.

\section{Method}

Subjects. Thirty-six proficient Chinese-English bilinguals were recruited in the present experiment. None of them had participated in the two previous experiments. Their general language background and L2 (English) proficiency were very much comparable to those of Experiments 1 and 2 subjects because (1) they had undergone the same language curriculum in high school; (2) they had generally the same language experience at home in terms of the predominantly monolingual environment of spoken language (i.e., Cantonese-Chinese); and (3) they had been required to demonstrate through examination a certain level of English proficiency before they could enroll at the university.

Materials and Apparatus. Materials and apparatus were the same as those in Experiment 1. New materials were two types of category names for use in the category-matching task: There were 2 remote categories (living and nonliving things) and 21 immediate categories (furniture, electrical appliances, means of transport, mammal, fruit, weapon, metallic instrument, stationery, organ, clothing, things to read, silverware, timer, plant, mechanical device, container, planet, musical instrument, building, signal, and vegetables). During category matching, immediate categories were assumed to demand fuller concept activation of the target than did more remote ones, due to their relatively high semantic specificity. The use of these two types of category names supposedly having different conceptual distances from any target items, therefore, provided an opportunity to assess the effects produced by different degrees of concept activation. The 21 immediate category names were generated by 10 independent subjects, who were asked to produce a most typical, first-come-to-mind superordinate category name for each of the 40 concepts underlying the 120 test items. Interjudge agreement on producing these 21 category names for the 40 concepts was higher than $84 \%$.

Procedure. The procedure of delayed production followed that used by Balota and Chumbley (1985), and it was identical to that of picture naming in Experiment 1, except that (1) words instead of pictures were presented and (2) the subject was required not to name the stimulus word as quickly as possible but to delay naming until a pair of parentheses came on bracketing the stimulus word. The parentheses always appeared $650 \mathrm{msec}$ after the onset of the test word (Balota \& Chumbley, 1985). Response latency was defined as the interval between the onset of the parentheses and the onset of the vocal response. This procedure was adopted in order to ensure a relatively pure measure of mere response production time, for processing, such as accessing the appropriate phonological code, and preparation for motor response should have been completed by the end of the 650 -msec delay.

For category matching, the subject was required to decide as quickly and accurately as possible whether the presented stimulus (picture, Chinese word, or English word) belonged to a superordinate category. The four factors (i.e., stimulus type, positive vs. negative match, language of the category name, and immediate vs. remote categories) were counterbalanced across concepts and subjects. Half of the stimuli in each block matched the category name. Each category-matching trial began with a square of arrays of small crosses. It was followed by a superordinate category name (English or Chinese), which was displayed for $1 \mathrm{sec}$. The stimulus (target) then followed immediately (i.e., SOA $=1,000 \mathrm{msec}$ ), and it stayed on the monitor until the subject responded by pressing either the designated yes or the designated no response key on the keyboard. No feedback was given. Category matching latency was defined as the interval between the onset of the target and the keypress.

\section{Results and Discussion}

Percent error. Error rates for both delayed production and category matching are presented in Table 3 . The only reliable difference was that between immediate and remote categories for the category-matching task $[F(1,117)=$ $\left.13.79, M S_{\mathrm{e}}=0.03, p<.001\right]$. Matching items to remote categories was in general more error prone than matching items to immediate categories.

Table 3

Mean Delayed Production and Category Matching Latencies (in Milliseconds; With Percent Error) for Experiment 3: Analysis by Subject

\begin{tabular}{|c|c|c|c|c|c|c|}
\hline \multirow[b]{3}{*}{ Task } & \multicolumn{6}{|c|}{ Target } \\
\hline & \multicolumn{2}{|c|}{ Chinese } & \multicolumn{2}{|c|}{ English } & \multicolumn{2}{|c|}{ Pictures } \\
\hline & $M$ & $S D$ & $M$ & $S D$ & $M$ & $S D$ \\
\hline Delayed production & 595 & 1.9 & 601 & 4.9 & & \\
\hline $\begin{array}{l}\text { Immediate category matching } \\
\text { (Chinese category names) }\end{array}$ & 971 & 7.1 & 1.093 & 7.9 & 956 & 9.2 \\
\hline $\begin{array}{l}\text { Immediate category matching } \\
\text { (English category names) }\end{array}$ & 1,068 & 7.8 & 1,229 & 8.1 & 1,129 & 8.8 \\
\hline $\begin{array}{l}\text { Remote category matching } \\
\text { (Chinese category names) }\end{array}$ & 1,154 & 13.8 & 1,370 & 21.7 & 1,099 & 12.5 \\
\hline $\begin{array}{l}\text { Remote category matching } \\
\text { (English category names) }\end{array}$ & 1,106 & 12.5 & 1,207 & 18.1 & 1,179 & 8.8 \\
\hline
\end{tabular}

Note-Only latencies of correct (and also positive for category matching) responses are reported. 
Latencies: Analyses by subject. Mean delayed production and category-matching latencies were calculated, using the subject as the unit of analysis. They are shown in Table 3. For delayed production, no effect of test language was found. For category matching, only the latencies of positive and correct responses were analyzed and used to indicate concept retrieval times. The justification was that a relatively pure concept access process could be assumed for positive and correct responses, whereas negative or incorrect responses might have involved other, additional processes. Category-matching latencies from positive and correct trials were analyzed by a 3 (Chinese, English, and picture stimuli) $\times 2$ (Chinese vs. English category names) $\times 2$ (immediate vs. remote categories) mixed design ANOVA. Category name was an insignificant factor, nor did it interact with any other effects. Both the immediate versus remote categories contrast $\left[F(1,34)=8.76, M S_{\mathrm{e}}=76,590.8, p=.006\right]$ and the effect of stimulus type $\left[F(2,68)=5.76, M S_{\mathrm{e}}=\right.$ $84,747.5, p=.005]$ were significant, though their interaction was not. The results indicated that there existed overall differences among category-matching times in response to picture, Chinese, and English items. More specifically, for immediate matching, English items were more slowly matched than both Chinese $[t(35)=-2.34$, $p=.02]$ and picture items $[t(35)=3.61, p=.001]$. Remote matching was slower for English than for Chinese items $[t(35)=-2.28, p=.03]$; the contrast between English and picture items was not significant. The shorter matching latencies associated with the Chinese than with the English items are indicative of stronger conceptual links for the former. The lack of a category name language effect is consistent with the results reported by Dufour and Kroll (1995), who also failed to find such effect in relatively fluent English-French bilinguals, as the current subjects were also considered proficient in their L2. Matching an item to a remote category was, in the present experiment, generally slower than matching it to an immediate category. This could be understood in terms of the differential category member priming relationships for immediate and remote categories. Immediate categories, because of their small numbers of members and, thus, close priming relationships with them, more strongly prime their members than do remote categories, which have weaker category member semantic relationships. Matching the target to a remote category would, therefore, take longer than matching it to an immediate one, due to the possibility that immediate category names more effectively prime the member names than remote category names do. This issue was not further pursued, because it fell out of the focus of the present study.

Latencies: Analyses by item. Mean delayed production and category-matching latencies were also calculated using the test item as the unit of analysis. As in the subject analysis, no effect of test language on delayed production latencies was found. Matching items to remote categories was slower than matching items to im- mediate categories $\left[F(1,114)=7.69, M S_{\mathrm{e}}=11,9391.0\right.$, $p=.006]$. No other effects were found reliable.

Retesting the hypotheses of Experiment 1. Delayed production and category-matching latencies were used as covariates in a set of ANCOVAs that reanalyzed the picture-naming and word translation data of Experiment 1 (simple correlations between picture-naming and word translation latencies and delayed production and category-matching times are shown in Appendix B). The reanalyses used the test item as the unit of analysis, because the covariates of delayed production and category matching were obtained from an independent subject group and were tied to individual test items. The reanalyses were driven by the previously tested hypotheses. First, it had been hypothesized that forward translation would be slower than backward translation. This comparison involved experimental conditions systematically differing in response language. Delayed production latency was, therefore, entered as a covariate. However, it only accounted for an insignificant portion of the translation time variance $\left[F(1,77)=0.19, M S_{\mathrm{e}}=81,705.7, p=\right.$ .667]. After the adjustment, forward translation was still significantly slower than backward translation $[F(1,77)=$ $\left.4.41, M S_{\mathrm{e}}=81,705.7, p=.04\right]$, with adjusted means of $1,374.1$ and 1,240.0, respectively. The first hypothesis was supported.

Second, it had been hypothesized that L1 picture naming would be faster than backward translation. This comparison involved experimental conditions systematically differing in stimulus type, and, thus, category-matching latencies were used as covariates. Neither immediate category-matching latency $\left[F(1,76)=0.02, M S_{\mathrm{e}}=\right.$ $42,432.9, p=.90]$ nor remote category-matching latency $\left[F(1,76)=1.18, M S_{\mathrm{e}}=42,432.9, p=.28\right]$ was a significant predictor. After the adjustment, $\mathrm{L} 1$ picture naming was still faster than backward translation $[F(1,76)=$ $\left.28.10, M S_{\mathrm{e}}=42,432.9, p<.001\right]$, with adjusted means of 992.0 and $1,244.5$, respectively. The second hypothesis was supported.

Third, it had been hypothesized that L2 picture naming would be no faster or slower than forward translation. This comparison involved experimental conditions systematically differing in stimulus type, and, thus, categorymatching latencies were used as covariates. Neither immediate category-matching latency $\left[F(1,76)=0.41, M S_{\mathrm{e}}=\right.$ $106,892.8, p=.53$ ] nor remote category-matching latency $\left[F(1,76)=0.47, M S_{\mathrm{e}}=100,892.8, p=.50\right]$ was a significant predictor. After the adjustment, L2 picture naming was no faster or slower than forward translation $[F(1,76)=$ $\left.1.96, M S_{\mathrm{e}}=100,892.8, p=.16\right]$, with adjusted means of $1,279.2$ and $1,378.9$, respectively. The third hypothesis was supported.

After adjusting for variations in response production and concept retrieval latencies, the present experiment obtained a pattern of results identical to that of Experiment 1 . Forward translation seemed to have involved processes different from those required by backward trans- 
lation, for the latencies associated with the two tasks differed from each other, even though the effect of response production had been statistically removed. L1-to-L2 communication appeared to involve both concept activation and L2 labeling, as it was as slow as L2 picture naming, which relied on a weak $\mathrm{L} 2$-concept connection. The short $L 1$ picture naming latencies, compared with backward translation times, indicated strong L1-concept links. Also, the category-matching data (analysis by the subject) revealed that the Chinese items were more closely linked to the conceptual memory than were the English items. These results are entirely compatible with the idea of asymmetry.

\section{GENERAL DISCUSSION}

The present study evaluated four hypotheses derived from the asymmetry model of bilingual memory proposed by Kroll and Stewart (1994), using stimulus items of very similar recognition thresholds and a set of procedures that took into account some item characteristics when testing the experimental effects. Forward translation was found to be slower than backward translation, even when the effect due to different response languages was partialled out. The translation time difference, nevertheless, was explainable by the item characteristic of familiarity. L2 picture naming took as long as forward translation, thus reinforcing the notion that $\mathrm{L} 1$-to- $\mathrm{L} 2 \mathrm{com}-$ munication had to be indirectly accomplished via a conceptual memory and was, thus, as slow as L2 picture naming, which was based on a weak conceptual path. L1 picture naming was faster than backward translation, probably because the former relied on a strong $\mathrm{L} 1$-concept link, whereas the latter involved a lexical path that was of lesser strength. The hypothetical asymmetry of conceptual processing was confirmed by the finding that matching Chinese items to categories was faster than matching English items. Overall, the present results are best accommodated by a version of asymmetry in which processing of verbal items in both lexicons could be conceptual. In normal conditions, however, $\mathrm{L} 1$ items are predominantly conceptually processed, whereas the processing of $\mathrm{L} 2$ items involves mainly direct, lexical level translations into their $\mathrm{Ll}$ equivalents (see Figure 1). This is confirmed by the relatively long forward translation latencies, as compared with backward translation latencies, found in Experiment 1 . The fact that $\mathrm{L} 1$ items are more quickly matched to category names than are L2 items (analysis by the subject) indicates stronger conceptual linkage for $\mathrm{L} 1$ than for $L 2$. In other words, the presentation of an $L 1$ item results in quicker and/or richer semantic activation than the presentation of an $\mathrm{L} 2 \mathrm{item}$. This conclusion is in line with the finding reported by de Groot et al. (1994) that the effect of semantic organization was observable in both forward and backward translation, though the former was affected more than the latter. Using the task of category matching, Dufour and Kroll (1995) also deduced that the bilingual could access the conceptual memory directly through both the $\mathrm{L} 1$ and the L 2 lexicon. The amount of semantic information being activated, however, differs for the two languages. Similar conclusions were drawn by La Heij et al. (1996).

Nevertheless, when item familiarity was taken into account and used as a covariate in the reanalysis, the difference between forward and backward translation times disappeared. One might, therefore, tentatively assume that familiarity has an effect on how verbal items are processed and translated in terms of the use of the various pathways specified by the asymmetry model. It could be that familiar verbal items, regardless of the language they are in, tend to be more closely associated with the conceptual memory than are unfamiliar ones. The translation of familiar L2 items would, therefore, involve more conceptual processing than that of unfamiliar L2 items. In this way, high item familiarity likens backward to forward translation in terms of the reliance on conceptual mediation, and, consequently, after translation latencies were adjusted for item familiarity, L1 and L2 items no longer activated internal processing mechanisms that were markedly different. Such interpretation seems probable, although Experiment 2 does not demonstrate any initial difference in familiarity between the Chinese and the English items presently used. Actually, the correlations between item familiarity and the outcome variables of forward and backward translation latencies obtained in the present study are very close to the corresponding figures reported by de Groot et al. (1994): The present correlation between $\mathrm{Ll}$ item familiarity and forward translation latencies was -.37 , whereas the corresponding figure in the de Groot et al. study was - .35; the present correlation between $\mathrm{L} 2$ item familiarity and backward translation latencies was -.50 and that reported by de Groot et al. was -.45 . Both studies established that (1) familiarity of the stimulus item facilitates word translation, as indicated by the negative correlations, and (2) such facilitation is more evident in backward than in forward translation, as the negative correlations were higher in the former task. This is consistent with La Heij et al.'s (1996) proposition of poorer conceptual activation and, therefore, greater sensitivity to semantic help (such as increased item familiarity) in backward than in forward translation; this is also in harmony with the asymmetrical structure put forward by Kroll and Stewart (1994) and Dufour and Kroll (1995).

Another objective of the present study was to analyze picture-naming and word translation times into components and to use an ANCOVA to statistically remove extraneous variations in concept retrieval and response production when testing hypotheses based on picturenaming and word translation data. The importance of such control is immediately obvious, yet no previous studies have ever attempted it. This study effectively rules out the possibility that picture-naming and word translation times, which supposedly reveal the bilingual verbal memory structure, would be "contaminated" by variations at the concept access and the response production stage. 
Category matching and delayed production were used to estimate concept access and response production times, respectively, and it was shown that variations in these estimated latencies played a rather insignificant role in affecting the pattern of picture-naming and word translation results. Although this is a null result in statistical terms (i.e., the covariates of delayed production and concept access times were insignificant), it still contributes to the literature by providing the information that, when using picture naming and word translation to explore the bilingual memory structure, test item differences in concept access and response production times within normal range are probably unimportant factors to consider.

One unique theoretical contribution of the present study is that it extends both the idea of asymmetry (Kroll \& Stewart, 1994) and the critical role played by test item attributes in bilingual processing (de Groot et al., 1994) to bilinguals speaking two very different languages. Previous studies in the area have used similar L1s and L2s, such as English and French (see, e.g., Dufour \& Kroll, 1995) and Dutch and English (see, e.g., de Groot et al., 1994), which collectively belong to the Indo-European family. Potter et al. (1984), Chen and Ho (1986), Chen and Leung (1989), and Chen (1990) did contrast Chinese with the European languages of English and French, but these studies did not look at asymmetry directly. This research confirms that (1) asymmetry also applies to bilinguals speaking the distinctive languages of Chinese and English and (2) the test item attribute of familiarity plays an important role in conditioning the lexical and conceptual processing among proficient Chinese-English bilinguals, thus reinforcing what de Groot et al. (1994) propose for Dutch-English bilinguals.

The present study also contributes to the literature in terms of methodology. The use of ANCOVAs in the present context allows direct testing of the asymmetry model and evaluation of the effects of potentially confounding variables to be done at the same time. Future research in the area should consider this to be a useful procedure for examining bilingual memory and its complex interactions with various factors, the effects of which are not always immediately obvious.

\section{CONCLUSION}

It is concluded from the present findings that the asymmetry model provides a useful framework for the study of the bilingual memory. The first language lexicon is more closely tied to an underlying conceptual memory than is the nonnative lexicon. Subjective ratings on the familiarity of stimulus items are correlated with word translation latencies; they can also explain statistically the difference in latencies between the two translation directions. This, however, does not constitute strong counterevidence against translation asymmetry. Rather, it is proposed that item familiarity conditions the activation of the processing pathways specified by the asymmetry model. The present study contributes to the literature by extending the applicability of asymmetry to two virtu- ally unrelated languages and by introducing a useful way to control for extraneous variations caused by test item attributes while testing the critical hypotheses. It also rules out the possibility that response production and concept access speeds contribute significantly to the pattern of picture-naming and word translation results, which has conventionally been used to tap into the bilingual memory structure.

\section{REFERENCES}

AlTARRIBA, J. (1990). Constraints on interlingual facilitation effects in priming in Spanish-English bilinguals. Unpublished doctoral dissertation, Vanderbilt University.

Altarriba, J., \& Mathis, K. M. (1997). Conceptual and lexical development in second language acquisition. Journal of Memory \& Language, 36, 550-568.

Balota, D. A., \& Chumbley, J. I. (1985). The locus of word-frequency effects in the pronunciation task: Lexical access and/or production? Journal of Memory \& Language, 24, 89-106.

CHEN, H.-C. (1990). Lexical processing in a non-native language: Effects of language proficiency and learning strategy. Memory \& $\mathrm{Cog}$ nition, 18, 279-288.

Chen, H.-C. (1992). Reading comprehension in Chinese: Some implications from character reading times. In H.-C. Chen \& O. J. L. Tzeng (Eds.), Language processing in Chinese (pp. 175-205). Amsterdam: Elsevier.

Chen, H.-C. (1996). Chinese reading and comprehension: A cognitive psychology perspective. In M. H. Bond (Ed.), Handbook of Chinese psychology (pp. 43-62). Hong Kong: Oxford University Press.

Chen, H.-C., Cheung, H., \& LAU, S. (1997). Examining and reexamining the structure of Chinese-English bilingual memory. Psychological Research, 60, 270-283.

CHEN, H.-C., \& Ho, C. (1986). Development of Stroop interference in Chinese-English bilinguals. Journal of Experimental Psychology: Learning, Memory, \& Cognition, 12, 397-401.

Chen, H.-C., \& Leung, Y.-S. (1989). Patterns of lexical processing in a nonnative language. Journal of Experimental Psychology: Learning, Memory, \& Cognition, 15, 316-325.

CHEN, H.-C., \& NG, M.-L. (1989). Semantic facilitation and translation priming effects in Chinese-English bilinguals. Memory \& Cognition, 17, 454-462.

DE Groor, A. M. B. (1992a). Bilingual lexical representation: A closer look at conceptual representations. In R. Frost \& L. Katz (Eds.), Orthography, phonology, morphology, and meaning (pp. 389-412). Amsterdam: Elsevier.

DE Groot, A. M. [B.] (1992b). Determinants of word translation. Journal of Experimental Psychology: Learning, Memory, \& Cognition, 18, 1001-1008.

de Groot, A. M. B., Dannenburg, L., \& van Hell, J. G. (1994). Forward and backward word translation by bilinguals. Journal of Memory \& Language, 33, 600-629.

Dufour, R., \& Kroll, J. F. (1995). Matching words to concepts in two languages: A test of the concept mediation model of bilingual representation. Memory \& Cognition, 23, 166-180.

Francis, W. N., \& Kǚ́rRA, H. (1982). Frequency analysis of English usage. Boston: Houghton Mifflin.

Hong Kong Department of Education (1986). A frequency count for words commonly used by Hong Kong junior secondary school students. Hong Kong: Hong Kong Government.

JARED, D., \& SEIDENBERG, M. S. (1991). Does word identification proceed from spelling to sound to meaning? Journal of Experimental Psychology: General, 120, 358-394.

Keatley, C. W., Spinks, J. A., \& de Gelder, B. (1994). Asymmetrical cross-language priming effects. Memory \& Cognition, 22, 70-84

Kroll, J. F., \& CURLEy, J. (1988). Lexical memory in novice bilinguals: The role of concepts in retrieving second language words. In M. Gruneberg, P. Morris, \& R. Sykes (Eds.), Practical aspects of memory (Vol. 2, pp. 389-395). London: Wiley.

Kroli., J. F., Dufour, R., Sholl, A., \& RoufCa, P. (1993. June). The 
role of concept mediation in acquiring a second language. Poster presented at the meeting of the American Psychological Society, Chicago.

KROLL, J. F., \& Sholl, A. (1992). Lexical and conceptual memory in fluent and nonfluent bilinguals. In R. J. Harris (Ed.), Cognitive processing in bilinguals (pp. 191-204). Amsterdam: Elsevier.

KROLL, J. F., \& STEWART, E. (1994). Category interference in translation and picture naming: Evidence for asymmetric connections between bilingual memory representations. Journal of Memory \& Language, 33, 149-174.

la Hei,, W., Hooglander, A., Kerling, R., \& van der Velden, E. (1996). Nonverbal context effects in forward and backward word translation: Evidence for concept mediation. Journal of Memory \& Language, 35, 648-665.

Noble, C. E. (1953). The meaning-familiarity relationship. Psychological Review, 60, 89-98.

Paivio, A., Yuille, J. C., \& Madigan, S. A. (1968). Concreteness, imagery, and meaningfulness values for 925 nouns. Journal of Experimental Psychology, 76(1, Pt. 2), 1-25.

Potter, M. C., So, K.-F., von Eckardt, B., \& Feldman, L. B. (1984).
Lexical and conceptual representation in beginning and proficient bilinguals. Journal of Verbal Learning \& Verbal Behavior, 23, 23-38. Schwanenflugel, P. J., Harnishfeger, K. K., \& Stowe, R. W, (1988). Context availability and lexical decisions for abstract and concrete words. Journal of Memory \& Language, 27, 499-520.

Sholl, A., Sankaranarayanan, A., \& KRoll, J. F. (1995). Transfer between picture naming and translation: A test of asymmetries in bilingual memory. Psychological Science, 6, 45-49.

SNODGRASs, J. G. (ED.) (1984). Concepts and their surface representations [Special issue]. Journal of Verbal Learning \& Verbal Behavior, 23(1).

SNodgrass, J. G., \& VANDERWART, M. (1980). A standardized set of 260 pictures: Norms for name agreement, image agreement, familiarity, and visual complexity. Journal of Experimental Psychology: Human Learning \& Memory, 6, 174-215.

Talamas, A., \& Kroll, J. F. (1993, February). Form-related errors in second language learning: A preliminary stage in the acquisition of L2 vocabulary. Paper presented at the Conference on Second Language Acquisition and Foreign Language Learning, Purdue University.

\section{APPENDIX A}

Simple Correlations Between Picture Naming, Word

Translation Latencies, and Relevant Item Variables

\begin{tabular}{llllll}
\hline & $\mathrm{FA}(\mathrm{P})$ & $\mathrm{CA}(\mathrm{P})$ & & & \\
L1 picture naming & -.35 & -.27 & & & \\
L2 picture naming & $-.53^{*}$ & $-.45 \dagger$ & & & \\
& $\mathrm{FA}(\mathrm{L} 1)$ & $\mathrm{CA}(\mathrm{L} 1)$ & $\mathrm{IM}(\mathrm{L} 1)$ & Freq(L1) & Phone(L1) \\
& -.37 & -.22 & -.11 & $-.39 \dagger$ & .27 \\
Forward translation & $\mathrm{FA}(\mathrm{L} 2)$ & $\mathrm{CA}(\mathrm{L} 2)$ & $\mathrm{IM}(\mathrm{L} 2)$ & Freq(L2) & Phone(L2) \\
& $-.50^{*}$ & -.33 & $-.38 \dagger$ & -.33 & .22 \\
\hline
\end{tabular}

Note-FA, familiarity; CA, context availability; IM, imageability; Freq, frequency; Phone, number of phonemes; P, pictures; L1, Chinese items; L2, English items. "Significant at .001. 'Significant at .01 .

\section{APPENDIX B}

Simple Correlations Between Picture-Naming and Word Translation Latencies and Delayed Production and Category-Matching Latencies

\begin{tabular}{lccc}
\hline & Delay(L1) & Imatch(P) & Rmatch(P) \\
L1 picture naming & -.05 & -.01 & .02 \\
& Delay(L2) & Imatch(P) & Rmatch(P) \\
L2 picture naming & .20 & -.04 & -.06 \\
& Delay(L2) & Imatch(L1) & Rmatch(L1) \\
Forward translation & .04 & .19 & -.12 \\
& Delay(L1) & Imatch(L2) & Rmatch(L2) \\
Backward translation & .16 & .03 & .17 \\
\hline
\end{tabular}

Note-Delay, delayed production; Imatch, immediate category matching; Rmatch, remote category matching; P, pictures; L1, Chinese items; L2, English items.

(Manuscript received June 27, 1996; 